\title{
Preoperative Sarcopenia is Associated with Poorer Compliance Rate of Adjuvant Chemotherapy and Worse Disease-free Survival of Patients with Stage $₫$ Colon Cancer
}

\section{Liang Yu}

Fujian Medical University Union Hospital

\section{Guangliang Chen}

Fujian Medical University Union Hospital

\section{Zongbin Xu}

Fujian Medical University Union Hospital

\section{Pan Chi}

Fujian Medical University Union Hospital

Zhifen Chen ( $\boldsymbol{V}$ chenzhifen@fjmu.edu.cn )

Fujian Medical University Union Hospital

\section{Research Article}

Keywords: Sarcopenia, colon cancer, adjuvant chemotherapy, prognosis

Posted Date: November 12th, 2021

DOI: https://doi.org/10.21203/rs.3.rs-1034158/v1

License: (c) (1) This work is licensed under a Creative Commons Attribution 4.0 International License.

Read Full License 


\section{Abstract}

Purpose: Preoperative sarcopenia has been proved to be associated with worse postoperative outcomes in cancer patients. This study aimed to evaluate whether preoperative sarcopenia affects the perioperative outcomes, adjuvant chemotherapy, and long-term outcomes of patients with stage $\nabla$ colon cancer.

Methods: Total 218 patients who underwent curative resection for stage $\otimes$ colon cancer in our department from January, 2015 and December, 2018 were retrospectively analyzed. Sarcopenia was assessed by total psoas index, which measured the total area the level of L3 vertebral body and normalized according to patients' height. Perioperative complications, postoperative adjuvant chemotherapy, and long-term prognosis were retrospectively analyzed.

Results: Of 218 patients, 100(45.9\%) patients were diagnosed with sarcopenia. Sarcopenia did not add the risk of perioperative complications $(20.0 \%$ vs $15.3 \%, P=0.357)$, but it increased hospital stays $(7.6 \pm 3.9$ vs $6.7 \pm 2.2$ days, $P=0.042)$. Patients with sarcopenia had a lower rate of receiving adjuvant chemotherapy ( $70.0 \%$ vs $82.2 \%, p=0.033$ ) and less likely to receive adequate adjuvant chemotherapy ( $58.6 \%$ vs 70.1 , $\mathrm{P}=0.08)$. Patients with adequate adjuvant chemotherapy had significantly better 3 -year OS $(89.8 \%$ vs $79.5, P=0.005)$ and a tendency of better 3-year DFS (76.4\% vs $63.6 \%, P=0.055)$ than those with inadequate adjuvant chemotherapy and Non-adjuvant chemotherapy. Compared with the patients without sarcopenia, patients with sarcopenia had significantly worse $3-y e a r$ DFS (76.9\% vs $62.8 \%$, $\mathrm{P}=0.026)$.

Conclusion: Preoperative sarcopenia was an important indicator to predict the compliance of AC in stage $\nabla$ colon cancer patients, and it is also a significant prognostic factor of worse 3-year DFS.

\section{Introduction}

Sarcopenia is defined as a progressive and generalized skeletal muscle disorder that is associated with an increased likelihood of adverse outcomes including falls, fractures, physical disability, and mortality by the European Working Group on Sarcopenia in Older People (EWGSOP) in its latest guideline. ${ }^{[1]}$ Sarcopenia can be exacerbated by the hypercatabolic state and inflammatory response caused by malignancy. ${ }^{[2]}$ Therefore, Sarcopenia has been proposed to be an alternative marker of frailty for cancer patients in some study groups, ${ }^{[3-5]}$ and preoperative sarcopenia is associated with poor postoperative outcomes, such as wound infection, anastomotic leakage, length of hospital stays, in patients with gastrointestinal surgery. ${ }^{[4,6]}$ There also were some studies finding sarcopenia was a negative prognostic factor for disease-free survival (DFS) and overall survival (OS) in colorectal cancer patients ${ }^{[7,8]}$.

Sarcopenia is commonly diagnosed by measuring cross-sectional skeletal muscle area at the level of the third lumbar(L3) on the preoperative CT scan in most studies. ${ }^{[4,5,9,10]}$ However, this method is often timeconsuming and complicated, and is not suitable for clinical application. Some studies tried to use the 
total psoas index (TPI), which measured the total area at the level of $L 3$ vertebral body and normalized according to patients' height, to assess sarcopenia and satisfactory results were obtained ${ }^{[8,11,12]}$. The TPI is easy to measure and reproduced in clinical practice.

The association between TPI and surgical outcomes has been explored in recent years, and the results indicated it was a poor prognostic predictor in colorectal cancer ${ }^{[11]}$. However, whether low TPI (sarcopenia) affected the compliance of patients to receive the recommended standard adjuvant chemotherapy (AC) has not been well explored. According to the guideline of the National Comprehensive Cancer Network (NCCN), patients with stage $₫$ colon cancer were recommended to receive AC because the results of multiple studies that demonstrated a clear benefits of $A C,{ }^{[13-16]}$ and the 6 months of oxaliplatin combined with either infusional 5-fluorouacil plus folinic acid (FOLFOX) or capecitabine (CAPOX) became standard adjuvant regimen because of a consistent, albeit moderate, disease-free survival benefit compared with that of fluorouracil plus folinic acid alone. ${ }^{[15,17]}$ However, the compliance rate of AC for patients with stage $₫$ colon cancer was unsatisfactory due to the complications, frailty after surgery, or economic reasons. Even in MOSAIC trial, only $74.7 \%$ patients completed the standard. ${ }^{[13]}$ Sarcopenia was associated with weakness and delayed recovery after surgery, which may be one important risk factor of the compliance of AC. The present study aimed to evaluate whether sarcopenia affected the compliance of patients to receive the recommended standard $A C$ and its impact on the long-term prognosis of stage $\triangle$ colon cancer.

\section{Materials And Methods Study population}

We retrospectively analyzed 1352 consecutive patients with localized colon cancer who underwent radical resection in the Department of Colorectal Surgery of Fujian Medical University Union Hospital (FMUUH) from January 2015 to December 2018. Patients who met the following criteria were included in

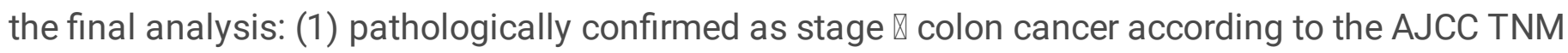
staging system; (2) received abdominal CT scan within 30 days before surgery; (3) received operation without neoadjuvant chemotherapy. Patients were excluded from the present study if they met any of the following criteria: (1) synchronous malignancy or a history of other malignant tumors; (2) distant metastatic diseases; (3) incomplete clinicopathological data. The flow chart was shown in Fig 1 . This retrospective study was approved by the institutional review board of FMUUH.

\section{Treatment and follow-up}

All the included patients underwent radical resection and were recommended to receive $A C$ according to the guideline of the National Comprehensive Cancer Network (NCCN) ${ }^{[18]}$ and the Ministry of Health of the People's Republic of China ${ }^{[19]}$. For p-Stage III colon cancer patients in our institute, the CAPOX (capecitabine and oxaliplatin) was a regular regime for AC. Adequate AC was defined as receiving CAPOX 
for more than 3 months. Inadequate AC included patients that received CAPOX less than 3 months and that only received oral capecitabine. The follow-up evaluations were performed every 3 months for the first 2 years, then every 6 months for the next 3 years, and annually thereafter. The postoperative followup included a physical examination, serum carcinoembryonic antigen (CEA) test, chest-to-pelvic CT, and colonoscopy. The baseline clinicopathological characteristics, postoperative complications (such as intestinal obstruction, anastomotic leak, abdominal infection, wound infection, pneumonia), recurrence status, and metastasis information of patients were collected from their medical records or through a telephone follow-up.

\section{Image analysis}

Total psoas area (TPA, $\mathrm{mm}^{2}$ ) was retrospectively measured on the preoperative CT scan at the level of the third lumbar vertebral (L3). The exact level of measurement was defined as the axial plane CT slice in which both L3 transverse processes were maximally in the view according to the earlier study. TPA was measured by manual outlining of both the left and right psoas muscle borders at the L3 level, and the area was automatically calculated on Picture Archiving and Communication System (PACS) software (Fig 2). The area was then normalized by the square of the height $\left(\mathrm{m}^{2}\right)$ to obtain the total psoas index (TPI, $\mathrm{mm}^{2} / \mathrm{m}^{2}$ ). The TPA was measured by two trained investigators (LY and GC) separately and the average was taken to final analysis, both were blinded to the patient outcomes at the time of quantification. Sarcopenia was defined using sex-specific thresholds of $<524 \mathrm{~mm}^{2} / \mathrm{m}^{2}$ for male patients and $<385 \mathrm{~mm}^{2} / \mathrm{m}^{2}$ for female patients according to earlier studies ${ }^{[8]}$. Sarcopenia was defined as an absolute variable and patients were classified as sarcopenia or non-sarcopenia in this study.

\section{Statistical analysis}

Categorical variables were present as frequencies and percentages, and Continuous variables were described as mean with standard deviation (SD) or interquartile range (IQR). Chi-square or Fisher's exact test was used to analyze categorical variables and a two-sample t-test or the Wilcoxon signed-rank test was performed to analyze continuous variables. The Kaplan-Meier method and the log-rank test were used for survival analysis. Multivariate analysis was conducted using a Cox proportional hazards model. All analyses were performed with SPSS 23.0 for MAC (SPSS Inc., Chicago, IL, USA). A two-tailed P value $<0.05$ was considered statistically significant.

\section{Results}

\section{Baseline demographic and clinicopathological characteristics}


A total of 218 eligible patients with stage $\nabla$ colon cancer were finally enrolled in this study between January 2015 and December 2018, including 135(61.9\%) males and 83(38.1\%) females, and the median age was 62 years (IQR 54-68 years). The median follow-up time was 34.3 months (IQR 26.6-51.3 months). The median TPI for all patients was $472.1 \mathrm{~mm}^{2} / \mathrm{m}^{2}$ (IQR $\left.364.5-581.6 \mathrm{~mm}^{2} / \mathrm{m}^{2}\right)$ and 549.0 $\mathrm{mm}^{2} / \mathrm{m}^{2}$ (IQR $\left.454.2-640.2 \mathrm{~mm}^{2} / \mathrm{m}^{2}\right), 357.8 \mathrm{~mm}^{2} / \mathrm{m}^{2}$ (IQR $\left.285.9-443.0 \mathrm{~mm}^{2} / \mathrm{m}^{2}\right)$. Baseline demographic and clinicopathological characteristics are shown in Table 1.

\section{Table 1}

Baseline demographics, clinicopathological characteristics of the patients with stage $\otimes$ colon cancer. 


\begin{tabular}{|c|c|c|c|c|}
\hline Characteristic & $\begin{array}{l}\text { All patients } \\
n=218(\%)\end{array}$ & $\begin{array}{l}\text { Patients with } \\
\text { sarcopenia } \\
n=100(\%)\end{array}$ & $\begin{array}{l}\text { Patients without } \\
\text { sarcopenia } \\
n=118(\%)\end{array}$ & $\begin{array}{l}P \\
\text { value }\end{array}$ \\
\hline Age $($ mean $\pm S D)$ & $60.9 \pm 12$ & $63.2 \pm 12.2$ & $58.9 \pm 11.5$ & 0.01 \\
\hline Gender & & & & 0.005 \\
\hline Male & $135(61.9)$ & $52(52.0)$ & $83(70.3)$ & \\
\hline Female & $83(38.1)$ & $48(48.0)$ & $35(29.7)$ & \\
\hline $\mathrm{BMI}\left(\mathrm{kg} / \mathrm{m}^{2}\right)$, mean $\pm \mathrm{SD}$ & $22.9 \pm 3.6$ & $21.6 \pm 3.3$ & $23.9 \pm 3.6$ & $<0.001$ \\
\hline Location of tumor & & & & 0.435 \\
\hline Left & $129(59.2)$ & $62(62.0)$ & $67 \rrbracket 56.8 \rrbracket$ & \\
\hline Right & $89(40.8)$ & 38(38.0区 & $51 \rrbracket 43.2 \rrbracket$ & \\
\hline pT & & & & 0.624 \\
\hline T1 & $2(0.9)$ & $1(1.0)$ & $1(0.8)$ & \\
\hline T2 & $2(0.9)$ & $0(0.0)$ & $2(1.7)$ & \\
\hline T3 & 172(78.9) & $79(79.0)$ & $93(78.8)$ & \\
\hline T4 & 42(19.3) & $20(20.0)$ & $22(18.6)$ & \\
\hline $\mathrm{pN}$ & & & & 0.403 \\
\hline N1 & 153(70.2) & 73(73.0) & $80(67.8)$ & \\
\hline N2 & $65(29.8)$ & $27(27.0)$ & $38(32.2)$ & \\
\hline pTNM & & & & 0.595 \\
\hline IIIA & $12(5.5)$ & $4(4.0)$ & $8(6.8)$ & \\
\hline IIIB & 160(73.4) & $76(76.0)$ & 84(71.2) & \\
\hline IIIC & $46(21.1)$ & $20(20.2)$ & $26(22.0)$ & \\
\hline Perineural invasion & & & & 0.037 \\
\hline Yes & $77(35.3)$ & $28(28.0)$ & $49(41.5)$ & \\
\hline No & $141(64.7)$ & $72(72.0)$ & $69(58.5)$ & \\
\hline Vascular invasion & & & & 0.035 \\
\hline Yes & 68(31.2) & $24(24.0)$ & $44(37.3)$ & \\
\hline No & $150(68.8)$ & $76(76.0)$ & $74(62.7)$ & \\
\hline ASA grade & & & & 0.811 \\
\hline
\end{tabular}




\begin{tabular}{|c|c|c|c|c|}
\hline 1 & $32(14.7)$ & 13(13.0) & 19(16.1) & \\
\hline 2 & $167(76.6)$ & $78(78.0)$ & $89(75.4)$ & \\
\hline 3 & $19(8.7)$ & $9(9.0)$ & $10(8.5)$ & \\
\hline In-hospital complication & & & & 0.357 \\
\hline Yes & $38(17.4)$ & $20(20.0)$ & 18(15.3) & \\
\hline No & $180(82.6)$ & $80(80.0)$ & $100(84.7)$ & \\
\hline Hospitalization days & $7.1 \pm 3.1$ & $7.6 \pm 3.9$ & $6.7 \pm 2.2$ & 0.042 \\
\hline Completion of AC & & & & 0.033 \\
\hline Adequate & $109(50.0)$ & $41(41.0)$ & $68(57.6)$ & \\
\hline Inadequate & $58(26.6)$ & $29(29.0)$ & $29(24.6)$ & \\
\hline Non-AC & $51(23.4)$ & $30(30.0)$ & 21(17.8) & \\
\hline $\mathrm{TPA}\left(\mathrm{mean} \pm \mathrm{SD}, \mathrm{mm}^{2}\right)$ & $1330.0 \pm 499.9$ & $980.7 \pm 310.5$ & $1626.0 \pm 435.0$ & $<0.001$ \\
\hline $\mathrm{TPI}\left(\mathrm{mean} \pm \mathrm{SD}, \mathrm{mm}^{2} / \mathrm{m}^{2}\right)$ & $472.1 \pm 165.8$ & $359.1 \pm 90.5$ & $597.4 \pm 133.2$ & $<0.001$ \\
\hline
\end{tabular}

The median interval between CT scan and operation was 9 days (IQR 6.0-14.0 days). Of these 218 patients, one hundred (45.9\%) patients were classified as sarcopenia by TPI cutoff values of male and female, including 52 (57.8\%) males and 48 (38.5\%) females. The ratio of sarcopenia in the female was higher than that in the male $(P=0.008)$. The average $B M I$ was $22.9 \mathrm{~kg} / \mathrm{m}^{2}(3.6)$, and patients with sarcopenia were more likely to have lower BMI than patients without sarcopenia $(21.6 \pm 3.3 \mathrm{vs}$ $\left.23.9 \pm 3.6 \mathrm{~kg} / \mathrm{m}^{2}, P<0.001\right)$.

\section{Postoperative outcome}

$38(17.4 \%)$ patients experienced postoperative complications after surgery. There was no significant difference in postoperative complications between sarcopenia and non-sarcopenia patients $(20.0 \% \mathrm{vs}$ $15.3 \%, P=0.357$ ). The mean length of postoperative hospital stay was 7.1 days ( $4-26$ days), and patients with sarcopenia had significantly longer postoperative hospital stays than those without sarcopenia (average $7.6 \pm 3.9$ vs $6.7 \pm 2.2$ days, $P=0.042$ ). Each group had one mortality during the hospital stay, one died of severe pneumonia and the other died of aspiration in the sarcopenia and non-sarcopenia group respectively.

\section{Adjuvant chemotherapy and survival analysis}


There were $76.6 \%$ of total patients receiving $\mathrm{AC}$, and $58.1 \%$ of them were non-sarcopenia. The rate of receiving $A C$ in the non-sarcopenia group was significantly better than the sarcopenia group $(82.2 \%$ vs $70 \%, p=0.033$, Figure $3 A$ ). Patients without sarcopenia had more likely to receive adequate AC (more than 3 months) than patients with sarcopenia ( $70.1 \%$ vs $58.6, P=0.084$, Figure $3 B)$, though the difference did not reach significance. It should be noticed that patients who received $A C$ had significantly better 3-year OS ( $87.6 \%$ vs $76.8 \%, P=0.007$, Figure $4 A$ ) and DFS ( $73.6 \%$ vs $59.5 \%, P=0.034$, Figure $4 B$ ) than patients without AC. In the subgroup analysis, when compared with patients who did not receive AC or received inadequate $A C$, patients who received adequate $A C$ had significantly better 3-year OS (89.8\% vs 79.5, $P=0.005$, Figure 4C) and a tendency of better 3-year DFS (76.4\% vs $63.6 \%, P=0.055$, Figure 4D).

The 3-year overall survival (OS) rate and disease-free survival (DFS) rate of total patients were $85.2 \%$ and $70.5 \%$, respectively. Patients with sarcopenia had significant worse 3-year DFS than that of patients without sarcopenia ( $76.9 \%$ vs $62.8 \%, P=0.026$, Figure $5 \mathrm{~A}$ ), although the 3 -year overall survival rate $(\mathrm{OS})$ of two teams had no significant difference $(90.0 \%$ vs $79.6 \%, P=0.092$, Figure $5 B)$.

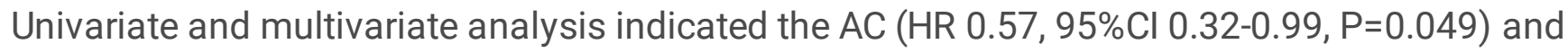
sarcopenia (HR 1.77, 95\% Cl 1.05-2.98, P=0.033) were significantly associated with 3-year DFS (Table 2).

Table 2

univariate and multivariate analysis of prognostic factor of 3-year DFS 


\begin{tabular}{|c|c|c|c|c|c|c|}
\hline \multirow[t]{2}{*}{ characteristics } & \multicolumn{3}{|c|}{ Univariate analysis } & \multicolumn{3}{|c|}{ Multivariate analysis } \\
\hline & $\begin{array}{l}\text { 3-year } \\
\text { DFS }\end{array}$ & $95 \% \mathrm{Cl}$ & $P$ value & Adjusted HR & $95 \% \mathrm{Cl}$ & $P$ value \\
\hline Age & & & 0.181 & & & \\
\hline$<65$ & $74.3 \%$ & $53.1-63.5$ & & & & \\
\hline$\leq 65$ & $65.1 \%$ & $45.9-59.2$ & & & & \\
\hline Gender & & & 0.216 & & & \\
\hline Male & $73.3 \%$ & 53.1-63.3 & & & & \\
\hline Female & $65.9 \%$ & $43.6-56.6$ & & & & \\
\hline BMI & & & 0.903 & & & \\
\hline$\geq 25$ & $72.8 \%$ & $48.2-63.7$ & & & & \\
\hline$<25$ & $69.6 \%$ & 49.7-59.3 & & & & \\
\hline Location of tumor & & & 0.528 & & & \\
\hline Left colon & $70.0 \%$ & $49.4-60.2$ & & & & \\
\hline Right colon & $71.3 \%$ & $51.1-63.9$ & & & & \\
\hline Pathological N stage & & & 0.002 & & & \\
\hline N1 & $78.2 \%$ & $55.8-65.1$ & & Ref & & \\
\hline N2 & $54.9 \%$ & $38.1-53.2$ & & 1.78 & $0.98-3.21$ & 0.057 \\
\hline Pathological TNM stage & & & 0.020 & & & \\
\hline IIIA & $90.9 \%$ & $54.8-76.7$ & & Ref & & \\
\hline IIIB & $74.7 \%$ & $53.4-62.7$ & & 0.27 & $0.03-2.17$ & 0.217 \\
\hline IIIC & $53.2 \%$ & $36.0-54.4$ & & 0.63 & $0.33-1.19$ & 0.150 \\
\hline Perineural invasion & & & 0.204 & & & \\
\hline Yes & $73.8 \%$ & $52.8-62.9$ & & & & \\
\hline No & $65.0 \%$ & $45.4-59.6$ & & & & \\
\hline Vascular invasion & & & 0.175 & & & \\
\hline Yes & $67.5 \%$ & 48.9-59.1 & & & & \\
\hline No & $76.7 \%$ & $52.5-66.7$ & & & & \\
\hline ASA stage & & & 0.636 & & & \\
\hline Q & $62.0 \%$ & $\begin{array}{r}\text { 40.9-63.1 } \\
\text { Page } 9\end{array}$ & & & & \\
\hline
\end{tabular}




\begin{tabular}{|c|c|c|c|c|c|}
\hline ૧ & $71.9 \%$ & $51.7-61.0$ & & & \\
\hline ૧ & $73.7 \%$ & 39.9-63.6 & & & \\
\hline In-hospital complication & & & & & \\
\hline Yes & $69.6 \%$ & $44.0-62.9$ & & & \\
\hline No & $70.7 \%$ & $51.3-60.4$ & & & \\
\hline Hospitalization days & & & & & \\
\hline$\geq 7$ days & $70.2 \%$ & $51.4-60.8$ & & & \\
\hline$<7$ days & $68.3 \%$ & $45.5-62.6$ & & & \\
\hline Adjuvant chemotherapy & & & & & \\
\hline No & $59.5 \%$ & $36.8-54.0$ & Ref & & \\
\hline Yes & $73.6 \%$ & $53.7-62.8$ & 0.57 & $0.32-0.99$ & 0.049 \\
\hline Sarcopenia & & & & & \\
\hline No & $76.9 \%$ & $54.7-65.4$ & Ref & & \\
\hline Yes & $62.8 \%$ & $42.6-54.5$ & 1.77 & $1.05-2.98$ & 0.033 \\
\hline \multicolumn{6}{|c|}{$\begin{array}{l}\mathrm{P} \text { value were calculated using the log-rank test for univariate analysis and COX proportional hazards } \\
\text { model for multivariate analysis. }\end{array}$} \\
\hline Ref: reference & & & & & \\
\hline
\end{tabular}

\section{Discussion}

This study showed that sarcopenia, which was defined by TPI, was significantly associated with a longer postoperative hospital stay in patients with stage $\otimes$ colon cancer who underwent laparoscopic radical resection, and patients with sarcopenia had a poorer completion rate of adequate AC. Sarcopenia was also a significant prognostic predictor for 3-year DFS.

Nowadays, there are two main methods to quantify sarcopenia, including measuring cross-sectional

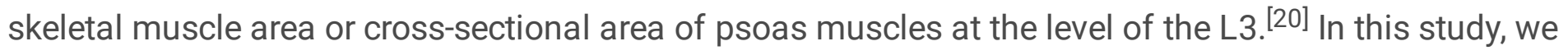
defined sarcopenia by measuring a sectional area of psoas muscles due to its operability and clinical practicality.

Sarcopenia was diagnosed in $19.6 \%$ to $32.9 \%$ of colorectal cancer patients in early studies. ${ }^{[8,11]}$ There were $45.9 \%$ of patients being classified as sarcopenia in our study. This may because we only included patients with stage $\otimes$ colon cancer, which was more likely to cause frailty. We found patients with sarcopenia had longer postoperative hospital stay than those without sarcopenia, though the overall rate 
of postoperative morbidity was not significantly different between the two groups. A longer postoperative hospital stay may be due to the frailty caused by sarcopenia, which made the patients need more time to recover from the surgical trauma.

In the past 30 years, $A C$ has been the standard treatment for patients with stage $\nabla$ colon cancer since the previous studies have shown the benefits in relapse-free survival and overall survival by using AC. ${ }^{[21,22]}$ Consistent with early studies, our study showed AC significantly improved 3-year OS and DFS. However, due to economic, physical, or psychological factors, not all patients with stage $\nabla$ colon cancer can finish the formal $\mathrm{AC}$ in clinical practice. ${ }^{[23]}$ Roger $\mathrm{H}$ et al found that up to $40 \%$ of patients with stage $\otimes$ or highrisk stage $\otimes$ colon cancer did not receive recommended AC in their study. ${ }^{[24]}$

According to the final results of a prospective, pooled analysis of six randomized, phase 3 trials (IDEA collaboration), the 5-year overall survival between 3 months and 6 months of $A C$ was similar $(82.1 \%$ versus $81.2 \%$, HR 0.96 [0.85-1.08]), which supported the use of 3 months of adjuvant CAPOX for most patients with stage III colon cancer. ${ }^{[21]}$ In our study, we define that receiving AC more than 3 months of CAPOX as an adequate AC. For the first time, we found sarcopenia was a significant factor to influence

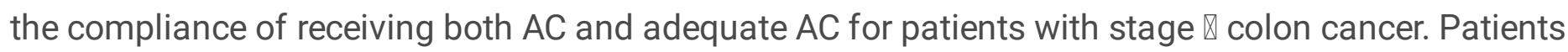
with sarcopenia had significantly lower compliance than those without sarcopenia. In addition, among patients who received $A C$, the rate of receiving adequate $A C$ in the non-sarcopenia group was higher than in the sarcopenia group.

The reason for a lower rate of adequate $\mathrm{AC}$ in the sarcopenia group may be that the surgery trauma aggravated the frailty of patients who had sarcopenia, which makes them unable to withstand the toxicity and complications of AC. Nowadays, some studies explored the feasibility and efficacy of neoadjuvant chemotherapy for locally advanced colon cancer and demonstrated the potential benefit when compared to $\mathrm{AC}^{[25-27]}$. Considering the low compliance of $\mathrm{AC}$ in patients with sarcopenia, neoadjuvant chemotherapy may be an option, and better nutrition support for the malnourished sarcopenia patients before the operation may benefit the short-term and long-term outcome, ${ }^{[28]}$ which need further study to confirm.

The prognostic significance of sarcopenia in patients with colorectal cancer (CRC) is controversial. A retrospective study of 220 stage $\rrbracket-\triangle C R C$ found that sarcopenia was an independent risk factor for both recurrence-free survival (RFS) and OS. ${ }^{[6]}$ However, another study including 494 patients with CRC showed sarcopenia did not significantly correlate with OS or RFS. ${ }^{[29]}$ The reason for the different results between the two studies may be that they used different diagnostic criteria. In this study, when using the TPI as the diagnostic criteria for sarcopenia, we found sarcopenia was a significantly negative prognosis factor of 3-year DFS in patients with stage $₫$ colon cancer. There was a tendency that patients with sarcopenia had poorer 3-year OS than those without sarcopenia ( $79.6 \%$ vs $90.0 \%, P=0.092)$, though the P-value did not reach the significance, the possible reason for these results is the relatively small sample size and inadequate follow up. 
There were some limitations of this study that should be addressed. First, this was a single-institution, retrospective study with relatively small sample size. Second, we used the predefined cutoff for sarcopenia in the previous study, which may be not appropriated for the population included in this study. The optimal cut-off values for TPI that define sarcopenia in Asian patients with colon cancer, still require further investigation. Third, limited by data, the dose intensity of chemotherapy drugs was not analyzed in this study, and whether the dose intensity influenced the completion rate of AC was not discussed.

\section{Conclusion}

To the best of our knowledge, this is the first study to explore whether sarcopenia affects the compliance of patients to receive the recommended AC. Sarcopenia was an important indicator to predict the compliance of postoperative $\mathrm{AC}$ of stage $\otimes$ colon cancer patients, and patients without sarcopenia had better compliance to the adjuvant chemotherapy. Sarcopenia was also a significant prognostic factor, which was associated with a worse 3-year DFS. To improve the compliance of AC and prognosis, it may be helpful to provide nutrition support and relieve sarcopenia before surgery, and further study on this topic is warranted. If possible, the more appropriate AC regimen should be administered according to the individual condition, and it can also further explore the feasibility of neoadjuvant chemotherapy in patients with sarcopenia.

\section{Declarations}

\section{Acknowledgement}

The authors would like to thank the advice and comments for this article from Prof. Ishihara, Dr. Nozawa, Dr. Kawai, Dr. Sasaki, and Dr. Murono of the Department of Surgical Oncology, The University of Tokyo Hospital, Japan.

\section{Conflicts of interest}

No author has any conflicts of interest related to this study.

\section{Funding}

This study was financially supported by the National Clinical Key Specialty Construction Project (General Surgery) of China (No. 2012-649). 


\section{References}

1. Cruz-Jentoft AJ, Bahat G, Bauer J, Boirie Y, Bruyere O, Cederholm T, et al. Sarcopenia: revised European consensus on definition and diagnosis. Age Ageing 2019;48:16-31. doi: 10.1093/ageing/afy169.

2. Prado CM, Wells JC, Smith SR, Stephan BC, Siervo M. Sarcopenic obesity: A Critical appraisal of the current evidence. Clin Nutr 2012;31:583-601. doi: 10.1016/j.clnu.2012.06.010.

3. Fearon K, Strasser F, Anker SD, Bosaeus I, Bruera E, Fainsinger RL, et al. Definition and classification of cancer cachexia: an international consensus. The Lancet Oncology 2011;12:489-495. doi: 10.1016/s1470-2045(10)70218-7.

4. Reisinger KW, van Vugt JL, Tegels JJ, Snijders C, Hulsewe KW, Hoofwijk AG, et al. Functional compromise reflected by sarcopenia, frailty, and nutritional depletion predicts adverse postoperative outcome after colorectal cancer surgery. Ann Surg 2015;261:345-352. doi:

$10.1097 /$ SLA. 0000000000000628.

5. Prado CMM, Lieffers JR, McCargar LJ, Reiman T, Sawyer MB, Martin L, et al. Prevalence and clinical implications of sarcopenic obesity in patients with solid tumours of the respiratory and gastrointestinal tracts: a population-based study. The Lancet Oncology 2008;9:629-635. doi: 10.1016/s1470-2045(08)70153-0.

6. Miyamoto Y, Baba Y, Sakamoto Y, Ohuchi M, Tokunaga R, Kurashige J, et al. Sarcopenia is a Negative Prognostic Factor After Curative Resection of Colorectal Cancer. Ann Surg Oncol 2015;22:2663-2668. doi: 10.1245/s10434-014-4281-6.

7. Choi MH, Oh SN, Lee IK, Oh ST, Won DD. Sarcopenia is negatively associated with long-term outcomes in locally advanced rectal cancer. J Cachexia Sarcopenia Muscle 2018;9:53-59. doi: 10.1002/jcsm.12234.

8. Dolan DR, Knight KA, Maguire S, Moug SJ. The relationship between sarcopenia and survival at 1 year in patients having elective colorectal cancer surgery. Tech Coloproctol 2019;23:877-885. doi: 10.1007/s10151-019-02072-0.

9. Lieffers JR, Bathe OF, Fassbender K, Winget M, Baracos VE. Sarcopenia is associated with postoperative infection and delayed recovery from colorectal cancer resection surgery. $\mathrm{Br} \mathrm{J}$ Cancer 2012;107:931-936. doi: 10.1038/bjc.2012.350.

10. Nozawa H, Emoto S, Murono K, Shuno Y, Kawai K, Sasaki K, et al. Change in skeletal muscle index and its prognostic significance in patients who underwent successful conversion therapy for initially unresectable colorectal cancer: observational study. Therap Adv Gastroenterol 2020;13:1756284820971197. doi: 10.1177/1756284820971197.

11. Richards SJG, Senadeera SC, Frizelle FA. Sarcopenia, as Assessed by Psoas Cross-Sectional Area, Is Predictive of Adverse Postoperative Outcomes in Patients Undergoing Colorectal Cancer Surgery. Dis Colon Rectum 2020;63:807-815. doi: 10.1097/DCR.0000000000001633. 
12. Murachi Y, Sakai D, Koseki J, Inagaki C, Nishida N, Yamaguchi T, et al. Impact of sarcopenia in patients with advanced or recurrent colorectal cancer treated with regorafenib. Int $\mathrm{J}$ Clin Oncol 2021;26:409-416. doi: 10.1007/s10147-020-01805-8.

13. André T, Boni C, Navarro M, Tabernero J, Hickish T, Topham C, et al. Improved overall survival with oxaliplatin, fluorouracil, and leucovorin as adjuvant treatment in stage II or III colon cancer in the MOSAIC trial. J Clin Oncol 2009;27:3109-3116. doi: 10.1200/jco.2008.20.6771.

14. Gray R, Barnwell J, McConkey C, Hills RK, Williams NS, Kerr DJ. Adjuvant chemotherapy versus observation in patients with colorectal cancer: a randomised study. Lancet 2007;370:2020-2029. doi: 10.1016/s0140-6736(07)61866-2.

15. André T, Boni C, Mounedji-Boudiaf L, Navarro M, Tabernero J, Hickish T, et al. Oxaliplatin, fluorouracil, and leucovorin as adjuvant treatment for colon cancer. N Engl J Med 2004;350:2343-2351. doi: 10.1056/NEJMoa032709.

16. Network ${ }^{\circledR}$ NCC. NCCN Clinical Practice Guidelines in Oncology. 2021;version 2. doi.

17. Haller DG, Tabernero J, Maroun J, de Braud F, Price T, Van Cutsem E, et al. Capecitabine plus oxaliplatin compared with fluorouracil and folinic acid as adjuvant therapy for stage III colon cancer. J Clin Oncol 2011;29:1465-1471. doi: 10.1200/jco.2010.33.6297.

18. Benson AB, 3rd, Bekaii-Saab T, Chan E, Chen YJ, Choti MA, Cooper HS, et al. Localized colon cancer, version 3.2013: featured updates to the NCCN Guidelines. J Natl Compr Canc Netw 2013;11:519-528. doi: 10.6004/jnccn.2013.0069.

19. Wang JP. [Chinese standard for the diagnosis and treatment of colorectal cancer (2010)]. Zhonghua Wei Chang Wai Ke Za Zhi 2011;14:1-4. doi.

20. Joglekar S, Nau PN, Mezhir JJ. The impact of sarcopenia on survival and complications in surgical oncology: A review of the current literature. J Surg Oncol 2015;112:503-509. doi: 10.1002/jso.24025.

21. André T, Meyerhardt J, Iveson T, Sobrero A, Yoshino T, Souglakos I, et al. Effect of duration of adjuvant chemotherapy for patients with stage III colon cancer (IDEA collaboration): final results from a prospective, pooled analysis of six randomised, phase 3 trials. The Lancet Oncology 2020;21:1620-1629. doi: 10.1016/s1470-2045(20)30527-1.

22. Levamisole and fluorouracil for adjuvant therapy of resected colon carcinoma. $\mathrm{N}$ Engl $\mathrm{J}$ Med 1990;323:197-198. doi: 10.1056/nejm199007193230312.

23. Xu Z, Mohile SG, Tejani MA, Becerra AZ, Probst CP, Aquina CT, et al. Poor compliance with adjuvant chemotherapy use associated with poorer survival in patients with rectal cancer: An NCDB analysis. Cancer 2017; 123:52-61. doi: 10.1002/cncr.30261.

24. Kim RH, Kavanaugh MM, Caldito GC. Laparoscopic colectomy for cancer: Improved compliance with guidelines for chemotherapy and survival. Surgery 2017;161:1633-1641. doi:

10.1016/j.surg.2016.11.024.

25. Cheong CK, Nistala KRY, Ng CH, Syn N, Chang HSY, Sundar R, et al. Neoadjuvant therapy in locally advanced colon cancer: a meta-analysis and systematic review. J Gastrointest Oncol 2020;11:847857. doi: 10.21037/jgo-20-220. 
26. Foxtrot Collaborative G. Feasibility of preoperative chemotherapy for locally advanced, operable colon cancer: the pilot phase of a randomised controlled trial. Lancet Oncol 2012;13:1152-1160. doi: 10.1016/s1470-2045(12)70348-0.

27. Karoui M, Rullier A, Piessen G, Legoux JL, Barbier E, De Chaisemartin C, et al. Perioperative FOLFOX 4 Versus FOLFOX 4 Plus Cetuximab Versus Immediate Surgery for High-Risk Stage II and III Colon Cancers: A Phase II Multicenter Randomized Controlled Trial (PRODIGE 22). Ann Surg 2020;271:637645. doi: $10.1097 /$ sla.0000000000003454.

28. Zhang T, Cao L, Cao T, Yang J, Gong J, Zhu W, et al. Prevalence of Sarcopenia and Its Impact on Postoperative Outcome in Patients With Crohn's Disease Undergoing Bowel Resection. JPEN J Parenter Enteral Nutr 2017;41:592-600. doi: 10.1177/0148607115612054.

29. Nakanishi R, Oki E, Sasaki S, Hirose K, Jogo T, Edahiro K, et al. Sarcopenia is an independent predictor of complications after colorectal cancer surgery. Surg Today 2018;48:151-157. doi: 10.1007/s00595-017-1564-0.

\section{Figures}




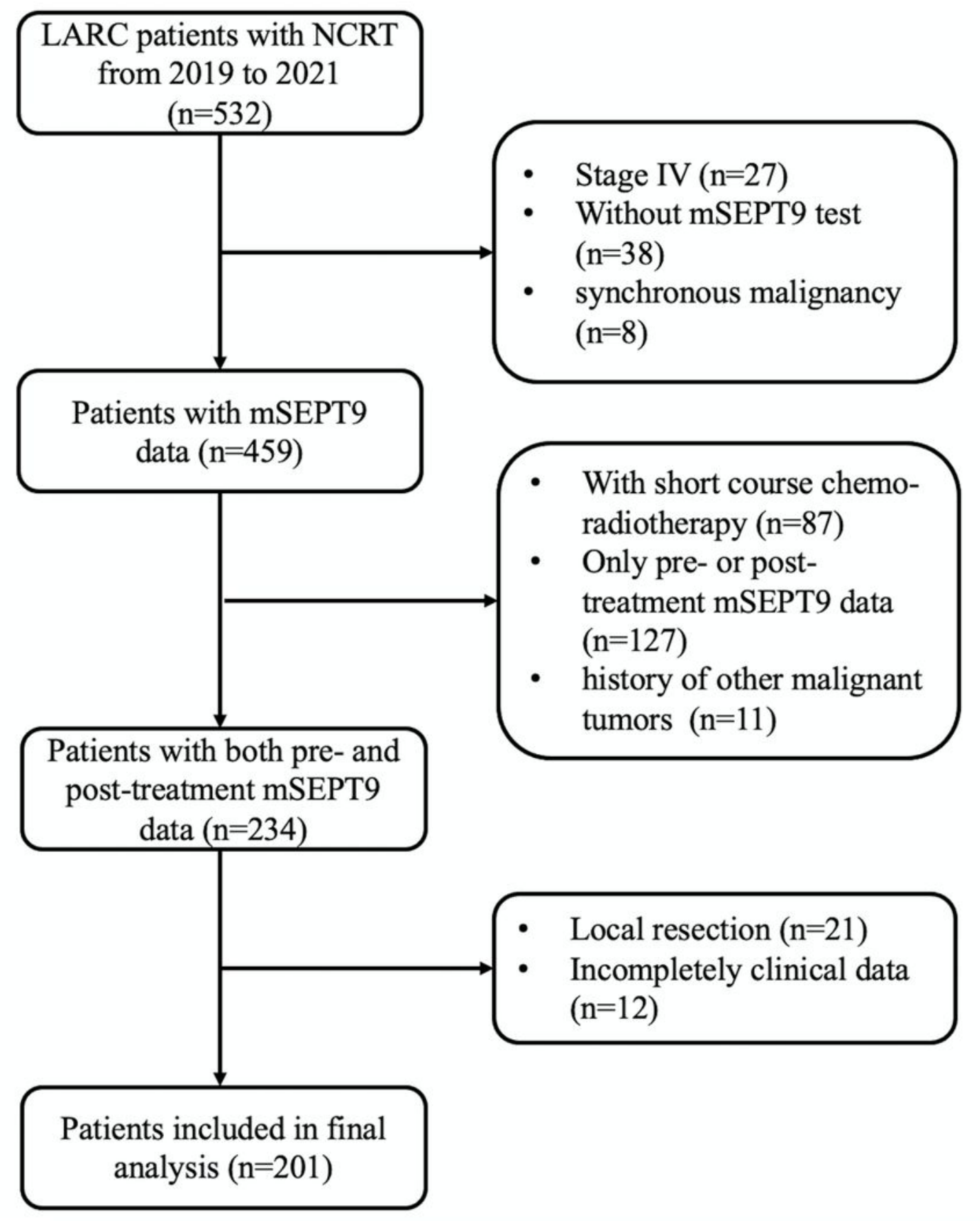

Figure 1

Flow chart. 


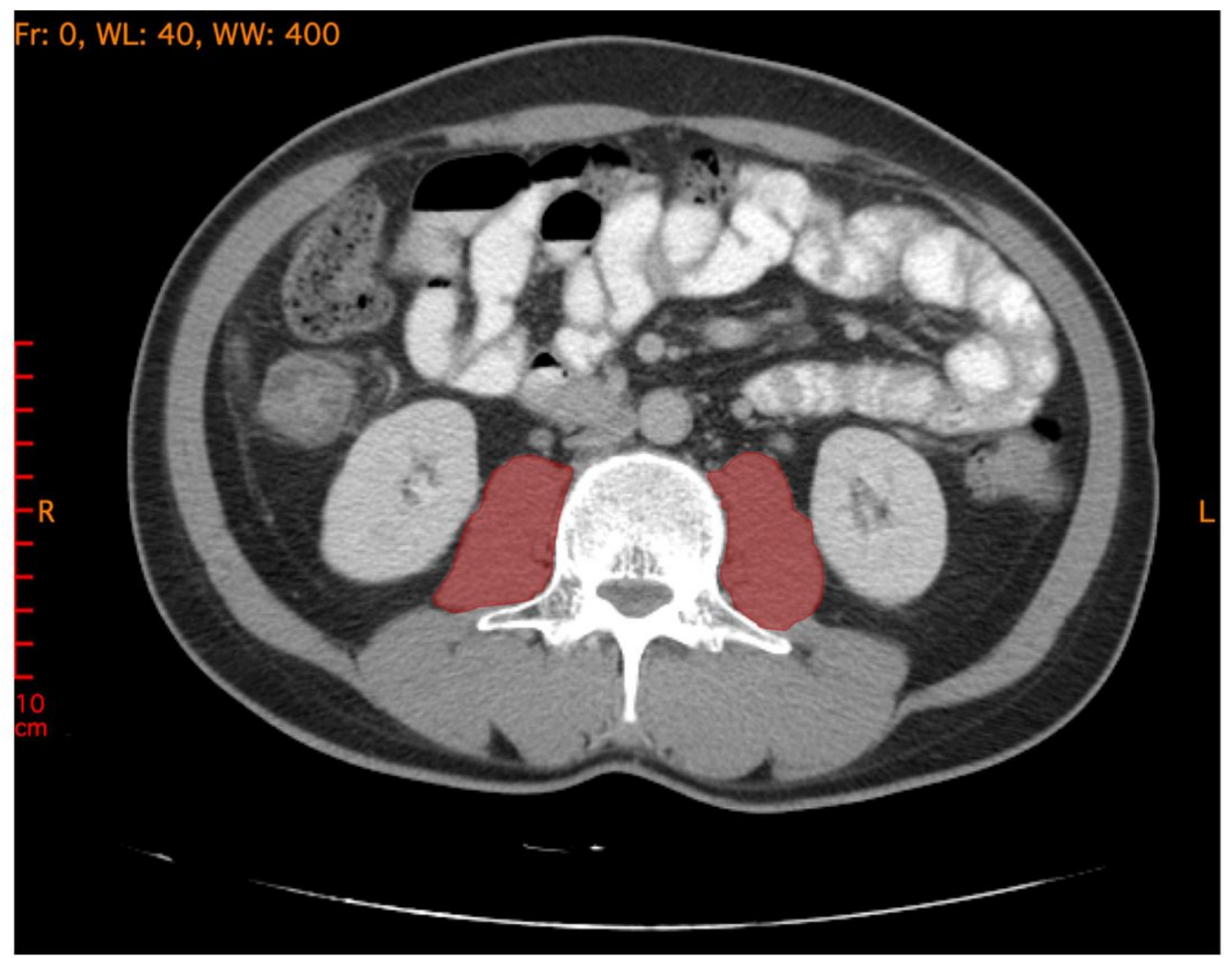

Figure 2

Psoas muscle (red area) at the level of L3 vertebral body. 

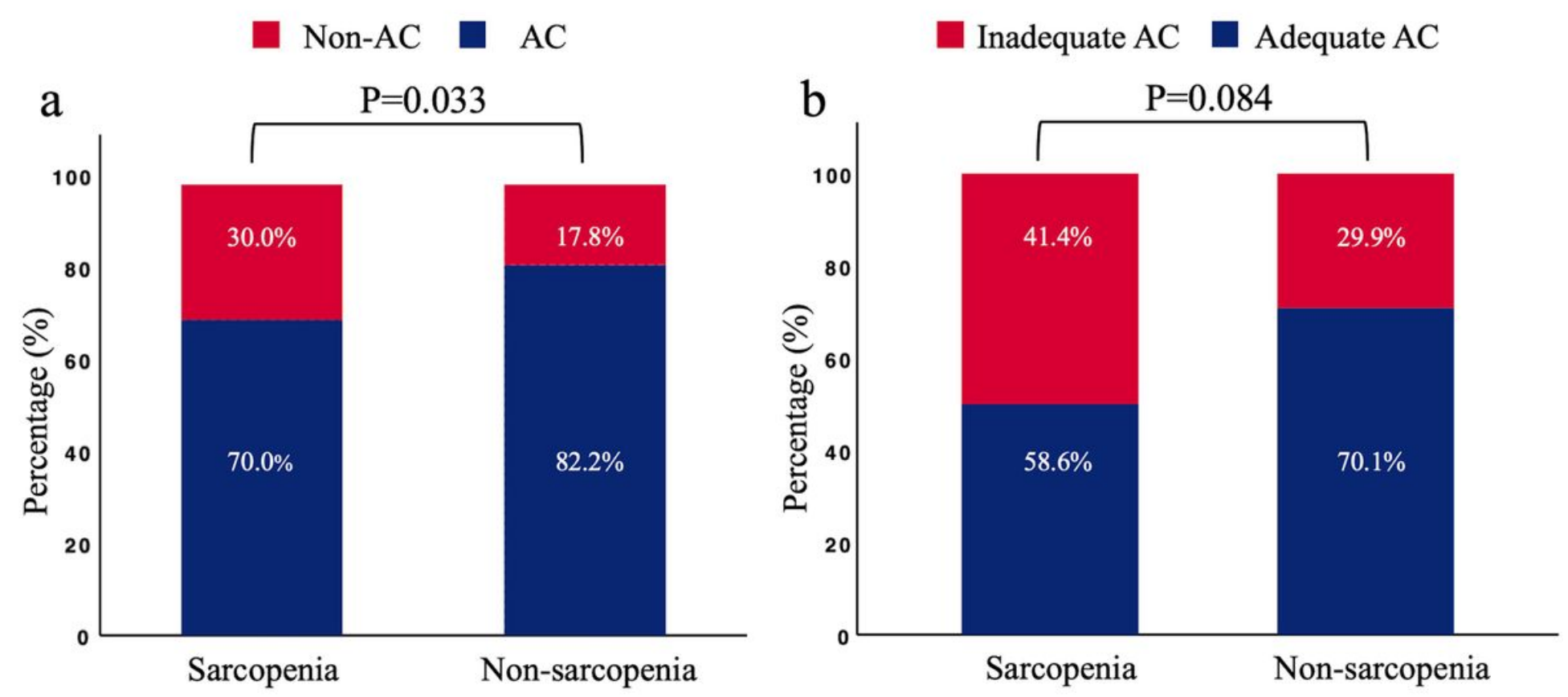

Figure 3

(A). The ratio of patients received adjuvant chemotherapy (AC) in sarcopenia group and non-sarcopenia group. (B). For all patients who received $A C$, the ratio of patients received adequate $A C$ and inadequate $A C$ in two groups. 

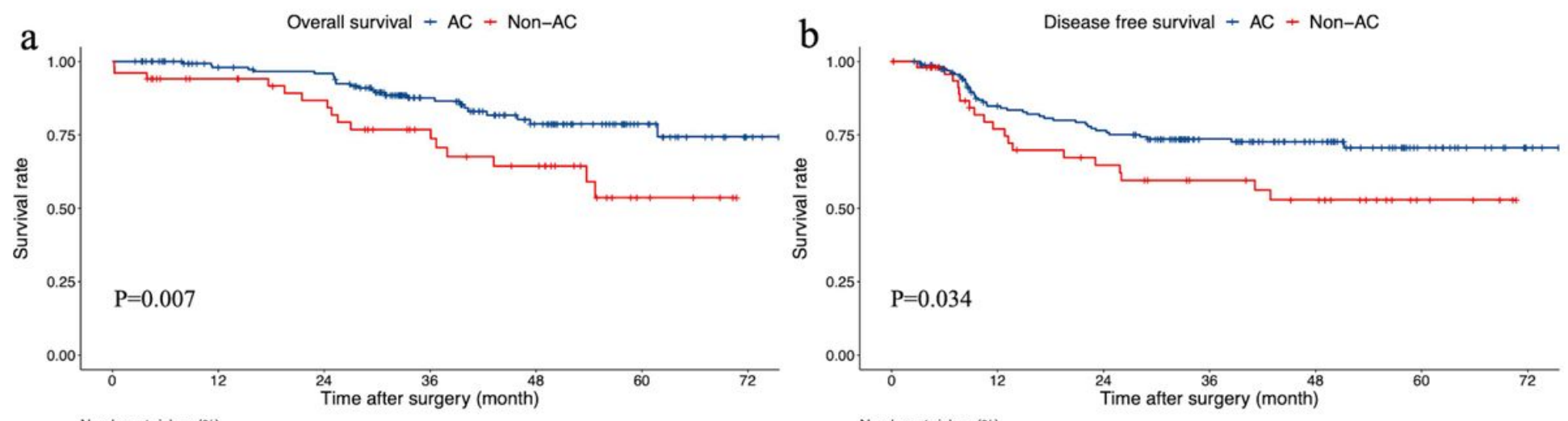

Number at risk: $\mathrm{n}(\%)$
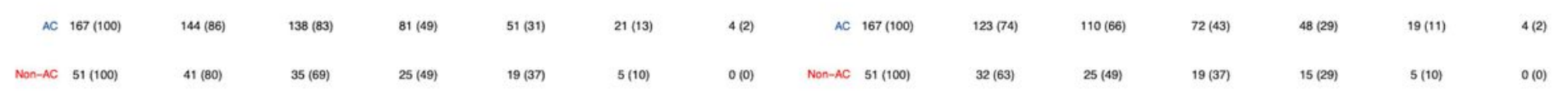

$\mathrm{c}$

Overall survival - Adequate AC - Inadequate AC \& Non-AC
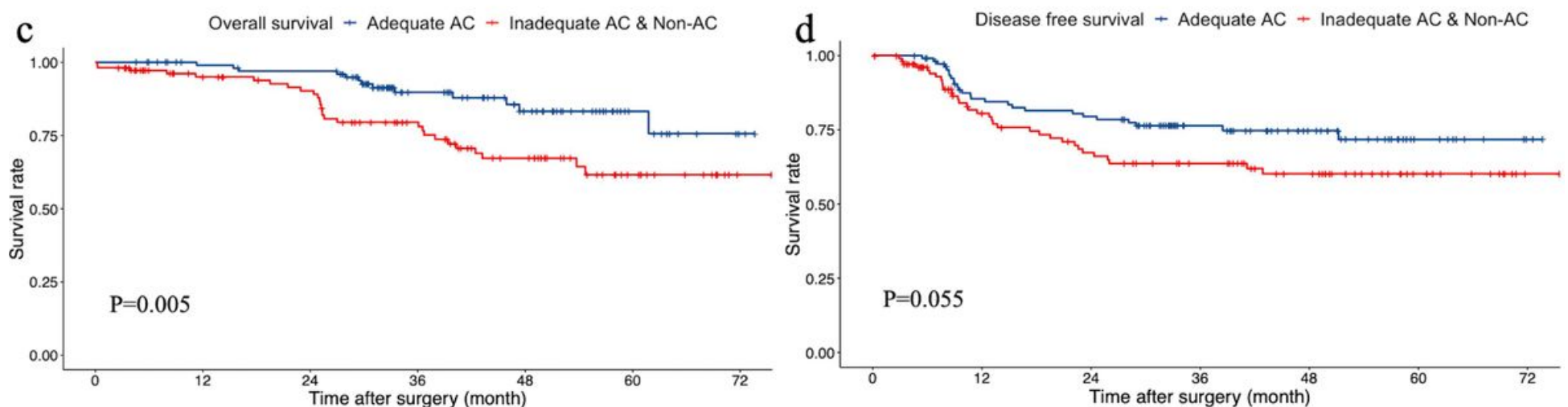

Number at risk: $\mathrm{n}(\%)$

\begin{tabular}{|c|c|c|c|c|c|c|c|}
\hline$A C>3$ & $109(100)$ & $100(92)$ & $97(89)$ & $51(47)$ & $33(30)$ & $11(10)$ & 2 (2) \\
\hline $\mathrm{ACS}$ & 109 (100) & 85 (78) & $76(70)$ & $55(50)$ & $37(34)$ & $15(14)$ & 2 (2) \\
\hline
\end{tabular}

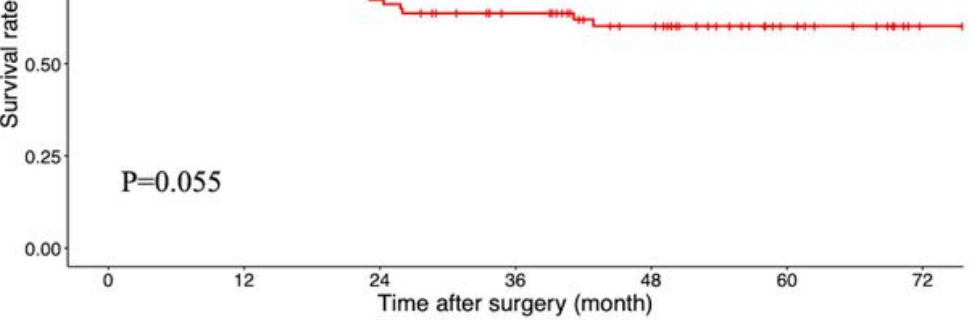
Number at risk: $\mathrm{n}(\%)$

\begin{tabular}{|c|c|c|c|c|c|c|c|}
\hline $\mathrm{AC}>3$ & $109(100)$ & $86(79)$ & $80(73)$ & $47(43)$ & 31 (28) & $10(9)$ & $2(2)$ \\
\hline 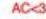 & $109(100)$ & $69(63)$ & $55(50)$ & $44(40)$ & 32 (29) & 14 (13) & $2(2)$ \\
\hline
\end{tabular}

\section{Figure 4}

(A). Analysis of overall survival according to whether patients received AC. (B). Analysis of disease-free survival according to whether patients received AC. (C). Patients who received adequate AC had significantly better 3-year OS than that received inadequate AC. (D) There was a tendency that Patients who received adequate AC had better 3-year DFS than that received inadequate AC. 

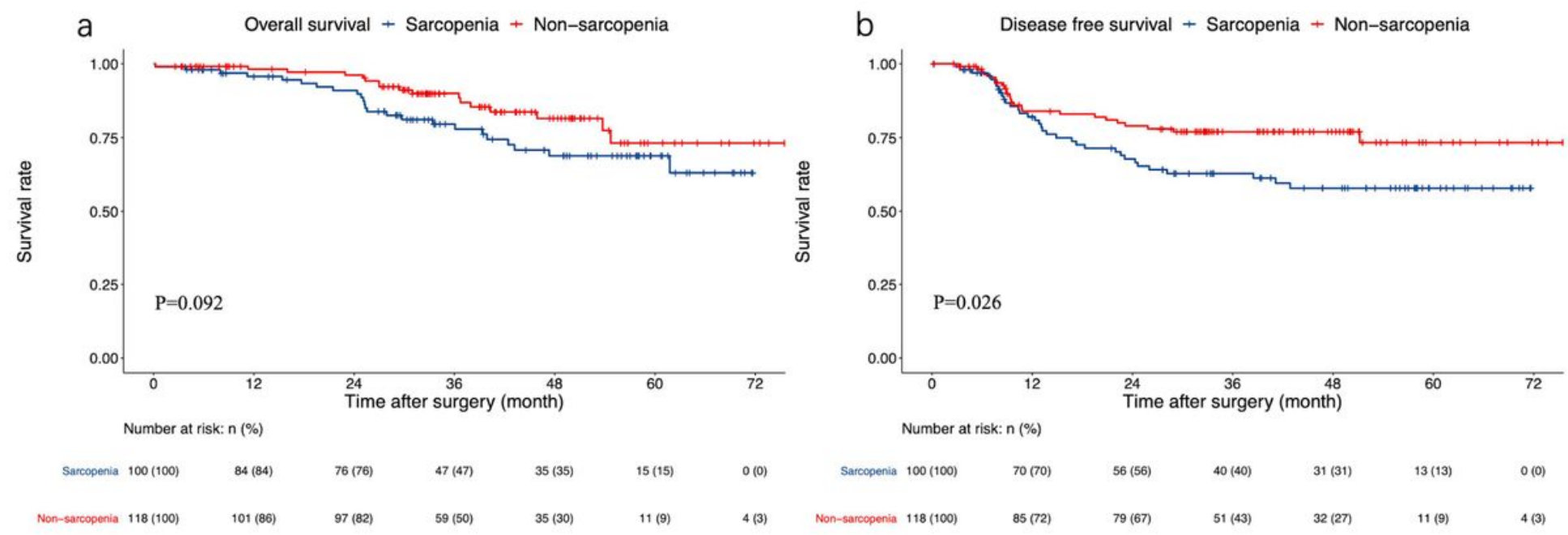

\section{Figure 5}

Analysis of overall survival (A) and disease-free survival (B) between patients with Sarcopenia and that without sarcopenia. 